subject of potentiation is not dealt with more fully and its relevance to crop protection discussed.

During the period between the publication of the fourth and fifth editions some major developments have occurred in the study of crop protection. In particular the need for a synthetic approach involving chemistry, physiology, population dynamies and genetics has become apparent. It is now generally accepted that much more attention should be directed to preventing the development of resistant strains of pests and to reducing undesirable side-effects of pesticides. The future of crop protection appears to lie with integrated control which is dependent on an ecological approach to the problem. Recent investigations on persistent organochlorine insecticides emphasize this need. These substances become distributed outside the area of application and become re-concentrated in food chains. Therefore they are particularly liable to favour the development of resistant strains in pests and to undermine biological control. These new developments are altering crop protection methods as well as the scientific principles on which they are based; it is disappointing that Dr. Martin has not discussed them in the fifth edition.

His book is still a valuable introduction of the chemical aspects of crop protection to the biologist, but it is not an adequate introduction of biological aspects to the chemist.

The new edition costs 25s. more than the fourth but is seventeen pages longer and much better produced. Quick reference to individual chemicals in the chapters on pesticides is now made easier by the use of bold type but is still a little impeded by the lack of headings to structural formulae.

N. W. MOORE

\section{ROLE OF ACETIC ACID IN BIOSYNTHESIS}

The Biosynthesis of Steroids, Terpenes and Acetogenins By Dr. John H. Richards and Dr. James B. Hendrickson. (Frontiers in Chemistry.) Pp. $x+416$. (New York and Amsterdam: W. A. Benjamin, Inc., 1964.) 20.35 dollars.

HE major pathways of biosynthesis to natural products are now reasonably well defined and in certain cases much detail is known concerning them. One of the most important of the biosynthetic pathways is that involving acetic acid, and The Biosynthesis of Steroids, Terpenes and Acetogenins describes its role in the biosynthesis of various natural products. These include the steroids and the terpenes together with a variety of other substances such as flavonoids, quinones, coumarins, chromones, etc., which are derived in part or in toto from acetic acid. While the generic terms, steroids and terpenes, are long established and well understood, no similar comprehensive term has hitherto been available for the other types of compounds produced from acetic acid. The authors have realized the necessity for such a term and have coined the word 'acetogenin' for this purpose.

This book had its origin in separate, shorter manuscripts produced by the authors. Dr. Hendrickson wrote about the acetogenins, while Dr. Richards described the terpenes and the steroids. Their original manuscripts were to constitute two chapters of a larger work on biosynthesis. For various technical reasons, this did not materialize, so the authors expanded their separate works and combined them. The present volume is the result of this synthesis of effort.

Chapters 1-5 inclusive are essentially the work of Dr. Hendrickson and are concerned with the role of acetic acid in the biosynthesis of the various types of acetogenin including fatty acids, aromatic compounds and flavonoids.
The correlation of many groups of natural products in terms of their derivation from acetic acid is admirably described together with the supporting experimental evidence where this is available.

The major contribution to this section consists of almost ninety pages detailing the statistical survey and the structural correlation of various natural products within the terms of their derivation from acetic acid. Much of this material has been described elsewhere, and I feel that this chapter could have been somewhat shortened with advantage, particularly since alternative, theoretical derivations of many compounds are described even when, in the following chapter, the known routes have been defined and the hypothetical alternatives excluded.

The remaining eight chapters of this book describe the biosynthesis of terpenes and steroids, starting from acetic acid and proceeding by way of mevalonolactone and the well-established routes to lanosterol and the various triterpenes. These chapters contribute a most valuable, concise statement of the relevant information in this field.

The book is well produced and the numerous formulae are printed with clarity and elegance. The adoption of Arabic rather than Roman numerals is to be commended. As is almost inevitable, a number of errors have crept in. For example, of the substances listed in the appendix under the heading "Xanthones", several, namely dehydrogriseofulvin, deschlorogriseofulvin, desmethyl-deschloro-griseofulvin, griseofulvin, goedin and erdin, are not xanthones: they are spirocoumaranones. There are also a number of minor errors in the references, but the principal source of comment in this connexion is the unfortunate number of errors in the formulae in the section concerning terpenes and steroids. For example, on p. 225, formula [1] for farnesyl pyrophosphate and formula [2] for nerolidol are both ineorrect. Formula [40] on p. 254 for rosenonolactone is devoid of a carbonyl group, while formula [41] on the same page for gibberellic acid has lost the carbonyl group of the lactonic function. A relatively frequent source of error in the formulae is the lack of appropriate methyl groups in the representations of many steroids and terpenoids. For example, in chart 1 on p. 334, several of the formulae have carbon methyl groups omitted. On p. 351, section (13-8) the formula on the left-hand side lacks the C-18 methyl group. On p. 354, the formula for progesterone lacks both the C-18 and the C-19 methyl groups. Methyl groups are similarly absont from the formula for germanicol on p. 272, formulae [11] and [15] on p. 243 , the upper formula in $(9-1)$ on p. 245, and the formula of cholest-5-ene on p. 308 . The statement on p. 364 that the compound funtumafrine $\mathrm{B}$ (formula [18]) possesses an oxidized C-18 angular methyl group is incorrect. Lophenol, formula [22] on p. 315, and methostenol, formula [23] on p. 316, are, of course, identical. Unfortunately, the formulae differ from each other and both are incorrect.

The lack of a unified system for the representation of the stereochemistry of the terpenes and steroids is disconcerting. This is particularly unfortunate in the discussions concerning derivatives of coprostane where, in general, no indication of the $\beta$ orientation of the $\mathrm{C}-5$ hydrogen atom is given.

Despite these defects, which can readily be remedied in a subsequent edition, this book is an extremely valuable contribution to the subject. It is, to the best of my knowledge, the first book adequately to cover the whole of the field of acetate biosynthesis. The publishers are to be congratulated for their courage in indicating the date of receipt of the manuscript together with the date of publication-a little more than twelve months. This book is extremely satisfactory value, even at the price, although this unfortunately will probably preclude its purchase by most private individuals. Its availability is a 'must', however, for all those interested in the field.

W. B. WhatTeY 